\title{
Particle damping with granular materials for multi degree of freedom system
}

\author{
Masanobu Inoue ${ }^{\mathrm{a}, *}$, Isao Yokomichi ${ }^{\mathrm{b}}$ and Koju Hiraki ${ }^{\mathrm{c}}$ \\ ${ }^{a}$ Department of Mechanical Engineering, Kitakyushu National College of Technology, Fukuoka, Japan \\ ${ }^{\mathrm{b}}$ Kitakyushu National College of Technology, Fukuoka, Japan \\ ${ }^{\mathrm{c}}$ Department of Mechanical and Control Engineering, Kyushu Institute of Technology, Fukuoka, Japan
}

Received 12 February 2010

Revised 3 August 2010

\begin{abstract}
A particle damper consists of a bed of granular materials moving in cavities within a multi degree-of-freedom (MDOF) structure. This paper deals with the damping effects on forced vibrations of a MDOF structure provided with the vertical particle dampers. In the analysis, the particle bed is assumed to be a single mass, and the collisions between the granules and the cavities are completely inelastic, i.e., all energy dissipation mechanisms are wrapped into zero coefficient of restitution. To predict the particle damping effect, equations of motion are developed in terms of equivalent single degree-of-freedom (SDOF) system and damper mass with use made of modal approach. In this report, the periodic vibration model comprising sustained contact on or separation of the damper mass from vibrating structure is developed. A digital model is also formulated to simulate the damped motion of the physical system, taking account of all vibration modes. Numerical and experimental studies are made of the damping performance of plural dampers located at selected positions throughout a 3MDOF system. The experimental results confirm numerical prediction that collision between granules and structures is completely inelastic as the contributing mechanism of damping in the vertical vibration. It is found that particle dampers with properly selected mass ratios and clearances effectively suppress the resonance peaks over a wide frequency range.
\end{abstract}

Keywords: Vibration suppression, particle damper, multi degree of freedom system, modal analysis, periodic motion

\section{Introduction}

The impact damper is an effective vibration absorber using a single mass free to move in a container mounted / integrated to a primary vibrating structure. Many theoretical, numerical, and experimental studies have been conducted for the characterization of impact damping effect [1-6]. Masri and Chaughey [2] carried out the first rigorous theoretical analysis of the dynamics and stability of the impact damper without gravity, using a coefficient of restitution to model the collisions between the mass and vibrating structure. They showed that in the immediate vicinity of resonance, the stability boundaries enclose a sufficient range of system parameters, such as coefficient of restitution, mass ratio, and clearance. Chatterjee et al. [5] analyzed the performance and design of an impact damper for attenuating the vibration of a Duffing oscillator with finite contact time impact model, where both elastic and inelastic collisions are considered. The optimum design was based around low amplitude, stable solution predicted by the harmonic balance method. In the case of completely inelastic vertical impact damper, where damper mass remains for some time in contact with the structure, Kaper [1] showed numerical results for both transient and forced vibration, where four possible phases of the impact vibration were distinguished, and good damping properties were obtained especially around resonance.

\footnotetext{
*Corresponding author. E-mail: masa@kct.ac.jp.
} 
A number of investigators have also studied the performance of multi-particle impact dampers (i.e., particle damper) [7-16]. This damper utilizes many particles within the damper container with particles having freely moving particles. Experiments in transient response have shown that particle dampers can be modeled with reasonable accuracy as single impact damper by treating the numerous impacting particles as one single particle with an effective coefficient of restitution [12]. An analytical study of the periodic motions of a particle damper applied to a vertical forced vibration has also been given, assuming a particle bed to be a single mass with coefficient of restitution of zero [16]. From the experiments, the model was found to accurately estimate the particle damping within the specified order of bed layers i.e., bed thickness, of granular particle.

In terms of particle damping mechanism, Fang and Tang [17] improved the analytical approximate modeling of granular damping using the multiphase flow theory [18] correlated with the numerical studies by discrete element method (DEM) [19,20]. They developed simulation studies for single DOF vertical forced vibration system, evaluating the interaction forces between the granules and the enclosure derived by the multiphase flow theory. Their case study shows that the particle damping with granular materials can yield substantial vibration suppression and parametric analysis can be greatly simplified by the new analytical model.

For the particle damping of beam and plate structures, on the other hand, $\mathrm{Xu}$ et al. [21,22] made experimental and numerical studies using a technique similar to DEM to characterize the effectiveness of the damping mechanism under random excitations. Their damping model due to the shear friction induced by strain gradient along the length of the structure was found to overestimate the damping compared to experiments. They also facilitated the development of application techniques for achieving high damping effect from the use of minimal quantity of particles. Indeed, DEM can lead to a quantitative understanding of the granular damping for wide range parameters, but involves a complicated dynamic model and highly time consuming computation. For a MDOF system subjected to collision / impact and sustained contact of particle beds distributed throughout MDOF structure, it is believed that an exact analysis of the periodic motion is very complicated.

In this report, therefore, we explore an approximate analysis for the periodic motion of the MDOF system provided with multiple particle dampers by developing equations of motion for its approximate SDOF system [16] by means of modal analysis. Numerical simulation studies were also carried out for the motion of the system by developing solutions valid between collision and contact of the damper mass. Experiments with a 3DOF structure using lead shot as a damper mass were conducted to corroborate the theoretical predictions. The effects of the number of damper units, container clearance, and mode shapes on the damping performance were considered.

\section{Equations of motion and analysis}

\subsection{Formulation}

The schematic model of the MDOF oscillating system equipped with the particle damper is shown in Fig. 1. The damper consists of a particle bed of mass $m_{d j}$ constrained to move vertically, with clearance $d_{j}$, in a cavity embedded in $j$-th vibrating body. In the analysis, the particle bed is assumed to be a mass which moves uni-diredtionally without friction and collides plastically with bottom / top or floor / ceiling of cavity. Thus the possible motions of the damper system can be divided into the two kinds of motion segments. In the sustained contact segment, the damper mass moves together with the primary mass after collision with end walls of cavity, while in the separate segment, the damper mass moves freely in the clearance.

The equation of motion for each segment can be written in the matrix form: these are, respectively, for separate segment (free flight phase)

$$
\begin{aligned}
& {[m]\{\ddot{x}\}+[c]\{\dot{x}\}+[k]\{x\}=\{f\} \quad \sin \omega t \quad \text { (primary body) }} \\
& m_{d j} \ddot{y}=-m_{d j} g \quad\left(j=1,2, \ldots, N_{d}\right) \quad \text { (damper mass) }
\end{aligned}
$$

and for sustained contact segment (riding phase)

$$
\begin{aligned}
& {\left[m_{c}\right]\left\{\ddot{x}_{c}\right\}+[c]\left\{\dot{x}_{c}\right\}+[k]\left\{x_{c}\right\}=\{f\} \sin \omega t-\left\{m_{d}\right\} g} \\
& \left(m_{c j j}=m_{j j}+m_{d j}\right) \quad \text { (primary body and damper mass) }
\end{aligned}
$$




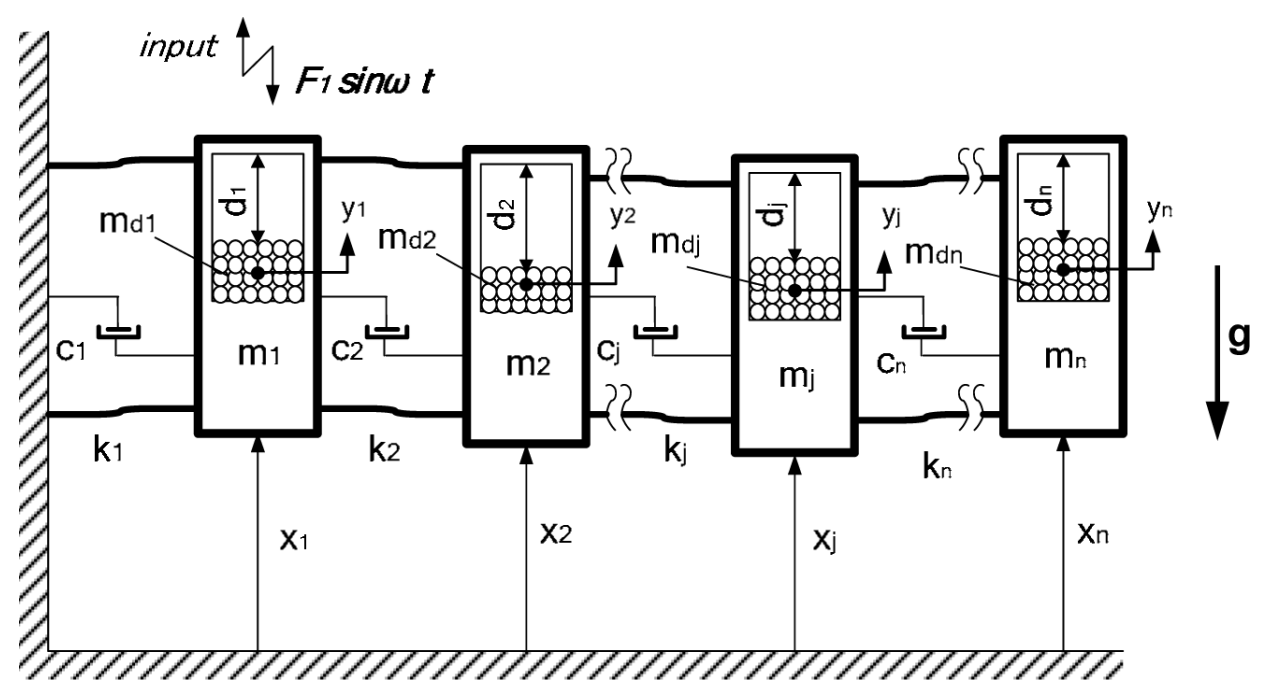

Fig. 1. MDOF system with particle damper.

where $[m],[c],[k]$ are the mass, damping, stiffness matrices, $\{x\}$ denotes displacement vector of the separate segment, $\{f\}$ the exciting force vector, and $\left\{x_{c}\right\}$ that of sustained contact segment, $\{f\}^{T}=$ $\left(0, \ldots, 0, f_{k}, 0, \ldots, 0\right),\left\{m_{d}\right\}^{T}=\left(m_{d 1}, \ldots, m_{d j}, \ldots, m_{d N_{d}}\right), t=$ time, $\omega=$ exciting frequency, and $g=$ acceleration of gravity. Applying the coordinate transformations

$$
\{x\}=[\phi]\{q\}, \quad\left\{x_{c}\right\}=\left[\phi_{c}\right]\left\{q_{c}\right\}
$$

where $[\phi]$ is the modal matrix, $\{q\}$ the normal coordinate for the separate segment, and $\left[\phi_{c}\right]$ and $\left\{q_{c}\right\}$ are those for the sustained contact segment, Eqs (1) and (2) are transformed, under proportional damping, into the form

$$
\begin{aligned}
& {[M]\{\ddot{q}\}+[C]\{\dot{q}\}+[K]\{q\}=[\phi]^{T}\{f\} \sin \omega t} \\
& {\left[M_{c}\right]\left\{\ddot{q}_{c}\right\}+\left[C_{c}\right]\left\{\dot{q}_{c}\right\}+\left[K_{c}\right]\left\{q_{c}\right\}=\left[\phi_{c}\right]^{T}\{f\} \sin \omega t-\left[\phi_{c}\right]^{T}\left\{m_{d}\right\} g}
\end{aligned}
$$

where $[M]\left(\left[M_{c}\right]\right),[K]\left(\left[K_{c}\right]\right)$ and $[C]\left(\left[C_{c}\right]\right)$ are diagonal matrices corresponding to the modal mass, stiffness, and damping matrices, respectively. The $i$-th mode equation of the system (4) and (5) are represented as follows:

$$
\begin{aligned}
& M_{i} \ddot{q}_{i}+C_{i} \dot{q}+K_{i} q_{i}=\phi_{k i} f_{k} \sin \omega t \\
& M_{c i} \ddot{q}_{c i}+C_{c i} \dot{q}_{c i}+K_{c i} q_{c i}=\phi_{c k i} f_{k} \sin \omega t-g \sum_{j=1}^{N_{d}} \phi_{c j i} m_{d j} \quad(i=1,2, \ldots, n)
\end{aligned}
$$

and their solutions, together with the motion of the damper mass $y_{j}$, are given by

$$
\begin{aligned}
& q_{i}=\exp \left(-\theta_{i} \tau\right)\left[A_{i} \sin \alpha_{i} \tau+B_{i} \cos \alpha_{i} \tau\right]+A_{p i} \sin \left(\tau-\beta_{i}-\varphi\right) \\
& q_{c i}=\exp \left(-\theta_{c i} \tau\right)\left[A_{c i} \sin \alpha_{c i} \tau+B_{c i} \cos \alpha_{c i} \tau\right]+A_{p c i} \sin \left(\tau-\beta_{c i}-\varphi\right)-\gamma_{i} \\
& y_{1 j}=-K_{R}\left(\tau-\tau_{0 j}\right)^{2} / 2+D_{1 j}\left(\tau-\tau_{0 j}\right)+E_{1 j} \quad \text { (upwards) } \\
& y_{2 j}=-K_{R}\left(\tau-\tau_{2 j}\right)^{2} / 2+D_{2 j}\left(\tau-\tau_{2 j}\right)+E_{2 j} \quad \text { (downwards) }
\end{aligned}
$$

where $\varphi$ is phase lag of exciting force with respect to impact at bottom, $\tau_{0 j}$ time of separation from bottom, and $\tau_{2 j}$ time of separation from top, and $\tau=\omega t$. The parameters of the $i$-th mode are given by $\omega_{i}=\sqrt{K_{i} / M_{i}}, \varsigma_{i}=$ $C_{i} / 2 M_{i} \omega_{i}, \theta_{i}=\varsigma_{i} / r_{i}, \alpha_{i}=\sqrt{\left(1-\varsigma_{i}^{2}\right)} / r_{i}, \quad r_{i}=\omega / \omega_{i}, K_{R}=g / \omega^{2}, \gamma_{i}=K_{R} r_{i} \mu_{i}, \delta_{i}=\phi_{k j} f_{k} / K_{i}, A_{p i}=$ $\delta_{i} / \sqrt{\left(1-r_{i}^{2}\right)^{2}+\left(2 \varsigma_{i} r_{i}\right)^{2}}, \tan \beta_{i}=2 \varsigma_{i} r_{i} /\left(1-r_{i}^{2}\right)$. The constants $A_{i}, B_{i}, A_{c i}, B_{c i}, D_{1,2 j}$ and $E_{1,2 j}$ are determined by the boundary conditions for each segment of motion. The complete behavior of the system, as given by Eqs (8) to (11) can be very complex, even for single-unit damper. The exact solutions for the periodic motion are difficult to obtain due to need of solving $2 N_{d}$ to $4 N_{d}$ nonlinear equations ( $N_{d}$ is the number of damper), corresponding to the type of periodic motion. Hence, a method based on approximating the system by an approximate SDOF system has been developed to study the damping effect of the impact damper on MDOF structure. 


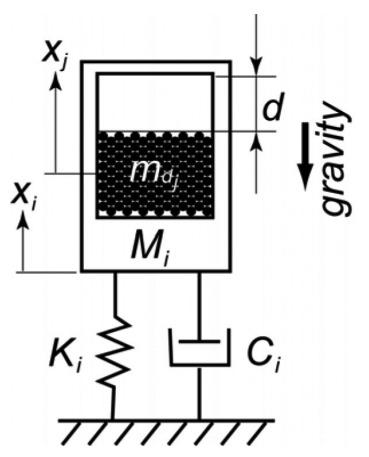

Fig. 2. Approximate SDOF model with particle damper for i-th mode.

\subsection{Approximate analysis}

Let us assume that the vibrating multibody system has well-separated resonances whose resonant peaks are primary concern of vibration reduction. Over relatively wide frequency ranges near natural frequencies, it can be represented as a SDOF model with parameters $M_{i}, C_{i}$, and $K_{i}$ (as shown in Eq. (6)), forced harmonically at resonant frequency $\omega_{i}$, as shown in Fig. 2. In order to suppress $i$-th mode vibration by applying the damper, it is necessary to estimate the modal mass $M_{i}$ so that it can represent the physically equivalent mass on the damper location. To this end, we normalize the modal vector $\left\{\phi_{i}\right\}$ associated with every one of the natural modes, with the value of the largest element of the vector equal to unity. The resulting modal parameters are given by

$$
\left[M_{i}\right]=[\bar{\phi}]^{T}[m][\bar{\phi}], \quad\left[K_{i}\right]=[\bar{\phi}]^{T}[k][\bar{\phi}], \quad\left[C_{i}\right]=\alpha\left[M_{i}\right]+\beta\left[K_{i}\right]
$$

where $[\bar{\phi}]$ is a modal matrix with normalized modal vectors $\left\{\bar{\phi}_{i}\right\}$ 's, and $\alpha$ and $\beta$ are constants used for specifying proportional-type damping.

Furthermore, in view of the fact that for a SDOF system provided with the particle damper, the damping effect varies with the acceleration level of the vibrating body (Araki et al.) [16], factors are involved in modifying the impacting mass ratio pertaining to the specified mode. Since the acceleration within the vibrating multibody system is proportional to the modal amplitude $\left\{\phi_{i}\right\}$, the effect of the damper location on the response of $i$-th mode can be approximately estimated by the total mode mass ratio

$$
\mu_{i}=\left(\sum_{j=1}^{N_{d}} \phi_{j i} m_{d j}\right) / M_{i}
$$

Thus, using the assumed mode approach retaining only one mode of the vibrating multibody system, the same analysis as the one developed for the SDOF system (Araki et al.) [16] can be applied to Eqs (6) and (7) to study the damper problem of the multibody system.

Among the major impact vibrations that exist in the vertical SDOF damper system are the following:

(1) Type I motion. The damper mass hits the floor alone either when the clearance is large enough damper mass not to hit the ceiling, or when the exciting level is low. The damper mass leaves the floor at time $\tau_{0}$, and then moves freely in the cavity until it collides with the floor without rebound (Fig. 3 (a)).

(2) Type II motion. Motion without sustained contact with the ceiling involves osculating contact. In this case, the damper mass detaches the ceiling at time $\tau_{1}$ just after collision with the ceiling (Fig. 3 (b)).

(3) Type III motion. The damper mass hits the ceiling at time $\tau_{1}$, and keeps contact with it until the acceleration reaches $-g$ at time $\tau_{2}$, i.e., sustained contact with the ceiling (Fig. 3(c)).

It remains now to find the steady-state solutions to the motions of the MDOF system subjected to the particle damper, using the approximate SDOF design technique.

The method for calculating the response of the approximate SDOF system to the particle damper is based on adjoining the successive motion segments over one cycle of the excitation by the condition of periodicity. To illustrate 


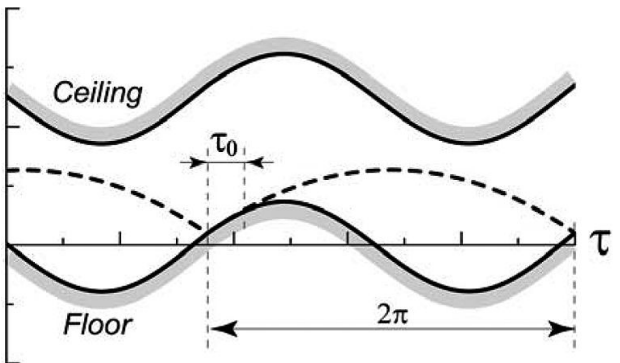

(a) Type I

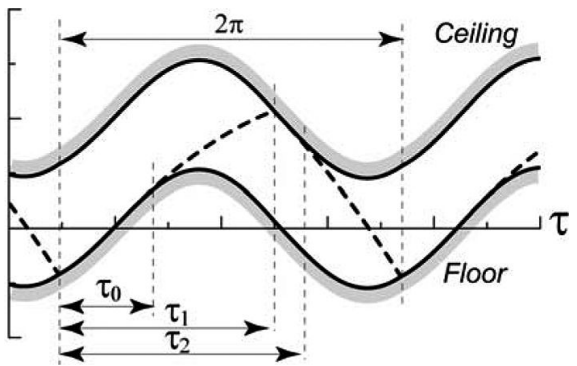

(c) Type III

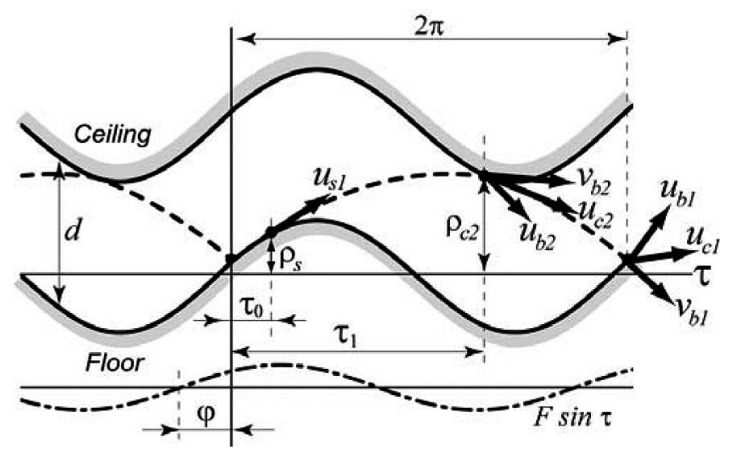

(b) Type II

Fig. 3. Type of vibration of SDOF system with particle damper.

the procedure, consider the periodic motion of Type II shown in Fig. 3(b), where the displacement and velocity of modal mass $M_{i}$ and damper mass $m_{d j}$ are shown for one period of the excitation. Due to the nature of the periodic motion, the solutions in Eqs (8)-(11) must satisfy the following conditions:

$$
\begin{aligned}
& \text { At } \tau=0 \quad: q_{c i}(0)=\rho_{c 1}, \quad q_{c i}^{\prime}(0)=u_{c 1}, \quad u_{c 1}=\left(\mu_{i} v_{b 1}+u_{b 1}\right) /\left(1+\mu_{i}\right) \\
& \text { At } \tau=\tau_{0} \quad: q_{c i}\left(\tau_{0}\right)=q_{i 1}\left(\tau_{0}\right)=y_{1 j}\left(\tau_{0}\right)=\rho_{s}, \quad q_{c i}^{\prime}\left(\tau_{0}\right)=q_{i 1}^{\prime}\left(\tau_{0}\right)=y_{1 j}^{\prime}\left(\tau_{0}\right)=u_{s} \\
& \text { At } \tau=\tau_{1} \quad: q_{i 1}\left(\tau_{1}\right)=q_{i 2}\left(\tau_{1}\right)=\rho_{c 2}, \quad y_{1 j}\left(\tau_{1}\right)=y_{2 j}\left(\tau_{1}\right)=\rho_{c 2}+d_{j}, \quad q_{i 1}^{\prime}\left(\tau_{1}\right)=u_{b 2}, \\
& y_{1 j}^{\prime}(\tau)=v_{b 2}, \quad q_{i 2}^{\prime}\left(\tau_{1}\right)=y_{2 j}^{\prime}\left(\tau_{1}\right)=u_{c 2}, \quad u_{c 2}=\left(\mu_{i} v_{b 2}+u_{b 2}\right) /\left(1+\mu_{i}\right) \\
& \text { At } \tau=2 \pi \quad: q_{i 2}(2 \pi)=y_{2 j}(2 \pi)=\rho_{c 1}, \quad q_{i 2}^{\prime}(2 \pi)=u_{b 1}, \quad y_{2 j}^{\prime}(2 \pi)=v_{b 1}
\end{aligned}
$$

Substituting Eqs (8)-(11) into the relations(14)-(17) leads to a set of three coupled algebraic equations in the form:

$$
\begin{aligned}
& \alpha_{c i} A_{c i}+\theta_{c i} B_{c i}+R_{1} A_{2 i}+R_{2} B_{2 i}+K_{R}\left(2 \pi-\tau_{1}\right) \mu_{i} /\left(1+\mu_{i}\right)=0 \\
& R_{3} A_{1 i}+R_{4} B_{1 i}+a_{45} A_{2 i}+a_{46} B_{2 i}-K_{R}\left(\tau_{1}-2 \pi\right)^{2} / 2-d_{j}=0 \\
& R_{5} A_{2 i}+R_{6} B_{2 i}-B_{c i}-K_{R}\left(2 \pi-\tau_{1}\right)^{2} / 2+d_{j}+K_{R} \mu_{i}=0
\end{aligned}
$$

together with the linear algebraic equation:

$$
\left[\begin{array}{cccccc}
0 & a_{12} & 0 & 0 & a_{15} & a_{16} \\
a_{21} & a_{22} & a_{23} & a_{24} & 0 & 0 \\
a_{31} & a_{32} & a_{33} & a_{34} & 0 & 0 \\
0 & 0 & a_{43} & a_{44} & a_{45} & a_{46} \\
a_{51} & a_{52} & 0 & 0 & 0 & 0 \\
0 & 0 & a_{63} & a_{64} & a_{65} & a_{66}
\end{array}\right] \quad\left[\begin{array}{c}
A_{c i} \\
B_{c i} \\
A_{1 i} \\
B_{1 i} \\
A_{2 i} \\
B_{2 i}
\end{array}\right]=\left[\begin{array}{l}
b_{1} \\
b_{2} \\
b_{3} \\
b_{4} \\
b_{5} \\
b_{6}
\end{array}\right]
$$


that determines the integral constants in Eqs (8) and (9). The remaining terms are available in Araki et al. [16].

Using the values of the specified modal parameters and employing numerical techniques for the solution of nonlinear algebraic Eqs (18)-(20), the values of $\tau_{0}, \tau_{1}$ and $\varphi$ can be determined. Then, the displacement of the modal mass $M_{i}$ can be found by evaluating $q_{i 1}(\tau), q_{i 2}(\tau)$ and $q_{c i}(\tau)$, respectively, for each motion segment.

\subsection{Simulation studies}

In view of the preceding approximate SDOF technique which requires the closed-form solution, an alternative approach to the exclusive reliance on analytical methods to estimate the response is to utilize simulation techniques. In motion of the vibrating MDOF system under action of the particle damper, there may occur impact vibrations other than those with the three types discussed previously. Simulations of the damped motion are performed to investigate the transient and steady-state response of the damper system by formulating the digital model.

Integrating the equations of motion (1) and (2) yields the following general solutions:

For separate segment $\left(\tau \geqslant \tau_{s j}\right)$ :

$$
\begin{aligned}
\left\{x_{s}\right\}= & {\left[G_{1}\left(\tau-\tau_{s j}\right)\right]\left\{x_{s 0}\right\}+\left[G_{2}\left(\tau-\tau_{s j}\right)\right]\left\{\dot{x}_{s 0}\right\} } \\
& -\left[G_{3}\left(\tau-\tau_{s j}\right)\right]\left\{h_{1}\left(\tau_{s j}\right)\right\}-\left[G_{4}\left(\tau-\tau_{s j}\right)\right]\left\{h_{2}\left(\tau_{s j}\right)\right\}+\left\{h_{1}(\tau)\right\} \\
y_{j}=- & K_{R}\left(\tau-\tau_{s j}\right)^{2} / 2+\dot{x}_{s j}\left(\tau-\tau_{s j}\right)+x_{s j}
\end{aligned}
$$

For sustained contact segment $\left(\tau \geqslant \tau_{c j}\right)$ :

$$
\begin{aligned}
\left\{x_{c}\right\}= & {\left[G_{1 c}\left(\tau-\tau_{c j}\right)\right]\left\{x_{c 0}\right\}+\left[G_{2 c}\left(\tau-\tau_{c j}\right)\right]\left\{\dot{x}_{c 0}\right\} } \\
& -\left[G_{3 c}\left(\tau-\tau_{c j}\right)\right]\left\{h_{1 c}\left(\tau_{c j}\right)\right\}-\left[G_{4 c}\left(\tau-\tau_{c j}\right)\right]\left\{h_{2 c}\left(\tau_{c j}\right)\right\}+\left\{h_{1 c}(\tau)\right\}
\end{aligned}
$$

where $\left(\left\{x_{s 0}\right\},\left\{\dot{x}_{s 0}\right\}\right)$ and $\left(\left\{x_{c 0}\right\},\left\{\dot{x}_{c 0}\right\}\right)$ are the initial conditions for the separate and contact segment, respectively, and $\tau_{s j}$ is time when $j$-th damper leaves the container end, $\tau_{c j}$ time at impact, and where

$$
\begin{aligned}
& {\left[G_{1}(\tau)\right]=[\phi][U(\tau)][Q], \quad\left[G_{2}(\tau)\right]=[\phi][V(\tau)][Q], \quad\left[G_{3}(\tau)\right]=[\phi][U(\tau)],} \\
& {\left[G_{4}\right]=[\phi][V(\tau)], \quad[Q]=[M]^{-1}[\phi]^{T}[m]}
\end{aligned}
$$

$[U],[V],\left\{h_{1}\right\}$ and $\left\{h_{2}\right\}$ are diagonal matrices and vectors with elements

$$
\begin{aligned}
& \left.u_{i i}(\tau)=\exp \left(-\theta_{i} \tau\right)\left[\left(\theta_{i} / \alpha_{i}\right) \sin \alpha_{i} \tau+\cos \alpha_{i} \tau\right)\right], \quad v_{i i}(\tau)=\exp \left(-\theta_{i} \tau\right)\left(1 / \alpha_{i}\right) \sin \alpha_{i} \tau \\
& h_{1 i}=A_{p i} \sin \left(\tau-\beta_{i}\right)-\gamma_{i}, \quad h_{2 i}=A_{p i} \cos \left(\tau-\beta_{i}\right) \quad(i=1,2, \ldots, n)
\end{aligned}
$$

and the undefined matrices and vectors with subscript $\mathrm{c}$ are those for the contact segment.

To proceed the computation of motion undergoing collisions and separations of the damper masses, it is necessary to evaluate the modal parameters, every time the damper masses change their respective phases of motion, that depend on the mass of the vibrating main body, varying spatially and temporally within the system. These modal parameters can be evaluated beforehand for $2^{N_{d}}$ possible combinations of motion phases just once and then stored for reuse. The calculation routine tests the phase of motion when any damper mass makes a transition from one phase to the next, and changes the current modal parameter to the one associated with a new combination of motion phase.

\section{Application}

The utility of the proposed approximate SDOF design technique is demonstrated by considering an example structure with a 3DOF system shown in Fig. 4(a) whose natural frequencies, mode shapes, and damping properties are obtained from experiments. Since in this case $n=3$, the natural frequencies are $\omega_{1}=81.9, \omega_{2}=218.8$, and $\omega_{3}=312.4[\mathrm{rad} / \mathrm{s}]$ and the corresponding three modal vectors are $\left\{\phi_{1}\right\}^{T}=\{0.415,0.783,1\},\left\{\phi_{2}\right\}^{T}=$ $\{1,0.498,-0.91\}$, and $\left\{\phi_{3}\right\}^{T}=\{-0.848,1,-0.465\}$, respectively. Figure 4(b) shows the frequency response curves without damper for three masses $m_{1}, m_{2}$, and $m_{3}$. 


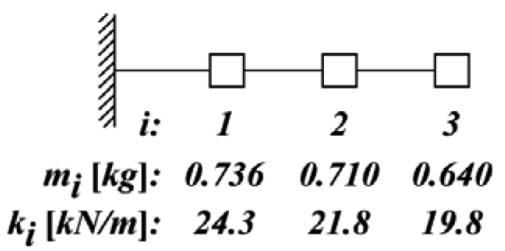

$(\alpha=1.55, \beta=1.4 E-5)$

(a) $3 \mathrm{DOF}$ system

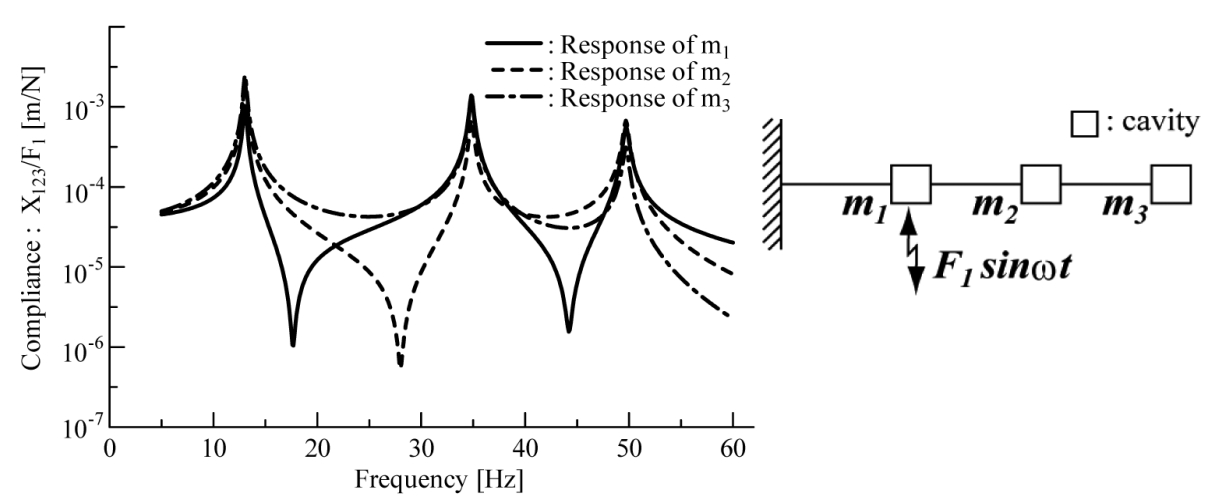

(b) Frequency response of Fig.4(a)

Fig. 4. Example structure.

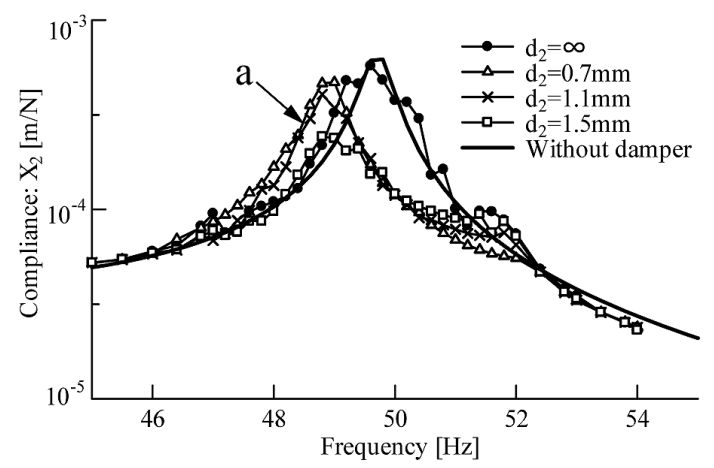

$\square$ : cavity with particle damper

(a) Single-unit damper at $\mathrm{m}_{2} . \quad\left(\mu_{d 2}=m_{d 2} / m_{2}=6 \%, F_{1}=5.8 \mathrm{~N}\right)$
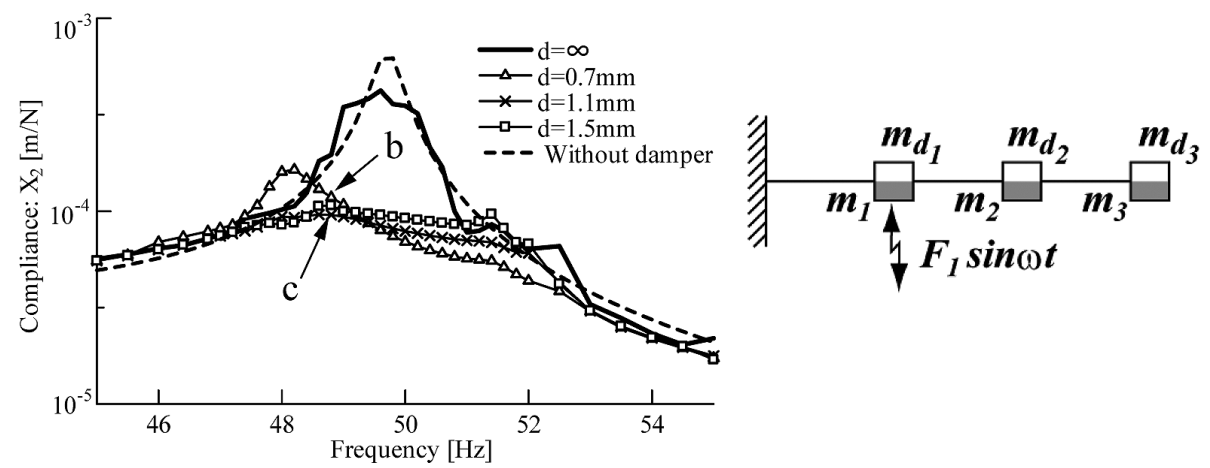

(b) Three-unit dampers at $\mathrm{m}_{1}, \mathrm{~m}_{2}, \mathrm{~m}_{3 .}\left(\mu_{d 1.2 .3}=m_{d i} / m_{i}=6 \%, F_{1}=5.8[N]\right)$

Fig. 5. Influence of the number of damper and clearance (Simulation). 


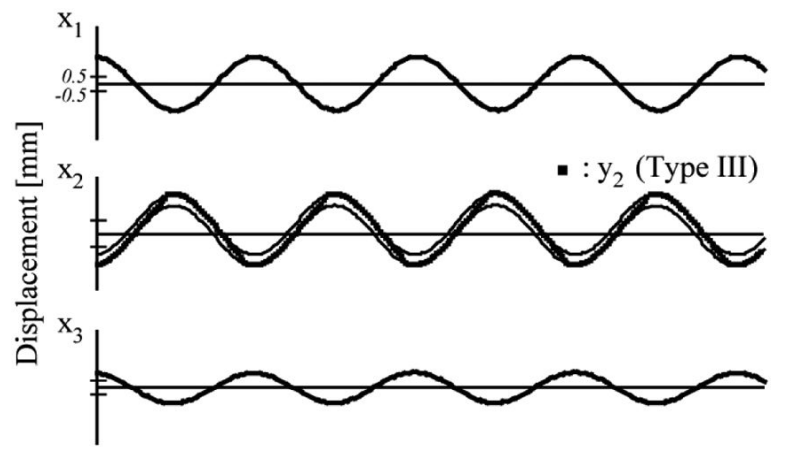

: cavity with particle damper

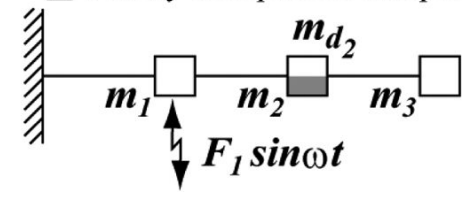

(a) Wave form at point "a" $\left(48.6[\mathrm{~Hz}], \mathrm{d}_{2}=0.7[\mathrm{~mm}]\right)$

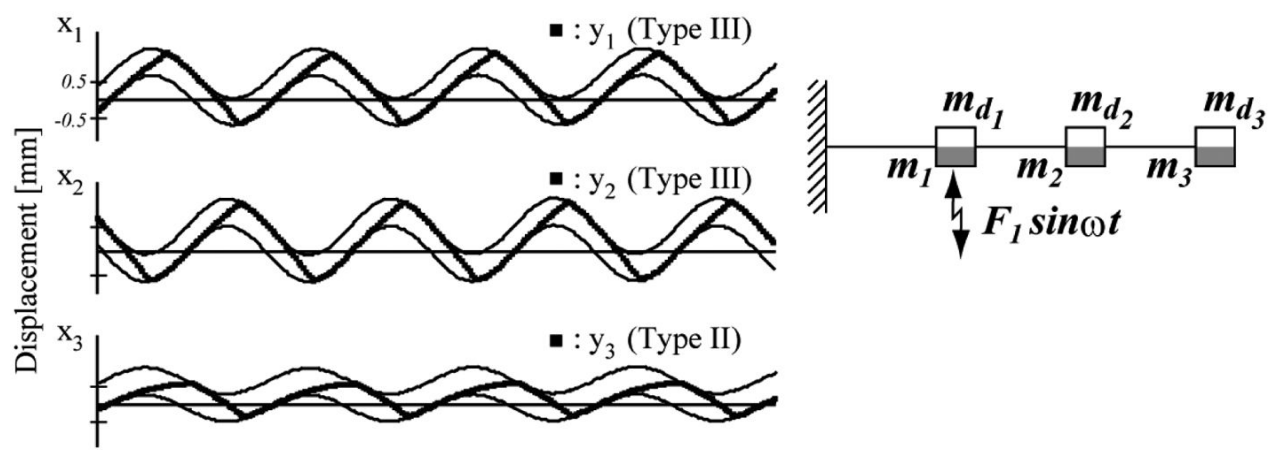

(b) Wave form at point "b" $(48.6[\mathrm{~Hz}], \mathrm{dj}=0.7[\mathrm{~mm}], \mathrm{j}=1,2,3)$

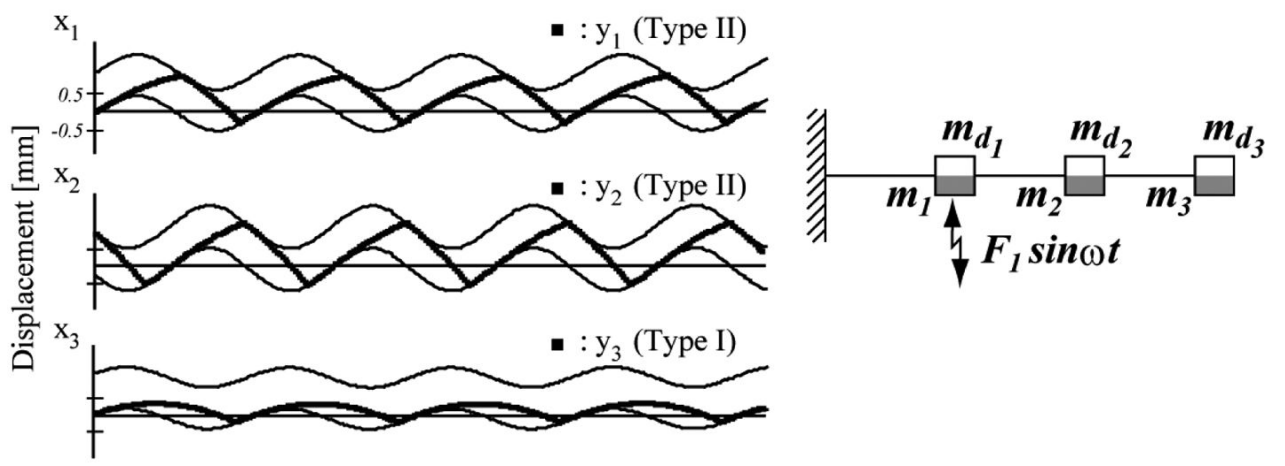

(c) Wave form at point "c" ( $48.6[\mathrm{~Hz}], \mathrm{dj}=1.1[\mathrm{~mm}], \mathrm{j}=1,2,3)$

Fig. 6. Type of impact vibration of MDOF system. (Simulation).

If this system is provided with single or three-unit particle dampers for properly selected clearance, the simulated frequency responses in its third mode are given by Fig. 5.

The displacement wave forms at the frequencies marked by a, b and c in Fig. 5 are shown in Fig. 6. In Fig. 6(a), a single damper is attached to mass $m_{2}$, i.e., a loop point, and in Fig. 6(b) three dampers are applied simultaneously to mass $m_{1}, m_{2}$, and $m_{3}$, respectively, with the identical clearance. The results in Fig. 5 show that what effects can be obtained by using plural dampers instead of the single unit damper, with all tuning and excitation parameters remaining the same. There are amplitude reductions around resonance by a factor $1 / 2.5$ to $1 / 4$ of the corresponding response of the single damper. The simulation wave forms indicate that three types of damper motions exist, and Type III motion shown in Fig. 6(a) enhances the motion of main mass by sustained contact of damper mass at both ends; while, for plural dampers, Type I and II motions effectively attenuate the resonant amplitude, as shown in Fig. 6(b) and 6(c). 

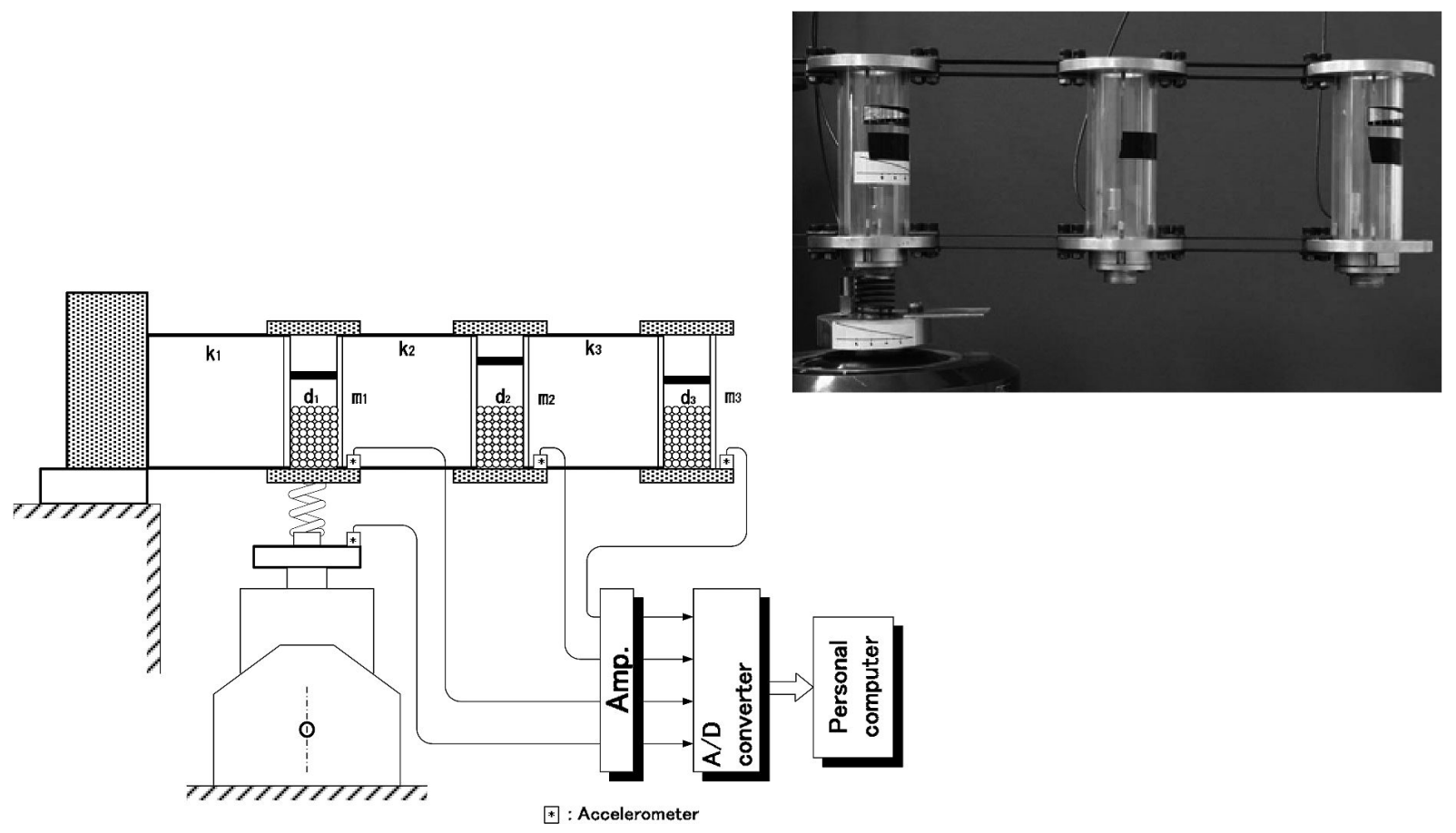

Fig. 7. Experimental setup for 3DOF structure.

\section{Experiments and discussion of results}

Experiments with particle dampers were conducted on a 3DOF model of the resonant structure to correlate the theoretical and experimental results. Figure 7 shows a construction of the apparatus and measurement system. Each main body incorporates a cavity of $40[\mathrm{~mm}]$ diameter by $120[\mathrm{~mm}]$ long with two flange ends, and it is supported by leaf springs. Using a screw mounted on the top flange, the clearance can be adjusted. The dimensions of the structures are: $m_{1}=0.74[\mathrm{~kg}], m_{2}=0.71[\mathrm{~kg}]$, and $m_{3}=0.64[\mathrm{~kg}] ; k_{1}=24.3[\mathrm{kN} / \mathrm{m}], k_{2}=21.8[\mathrm{kN} / \mathrm{m}]$, and $k_{3}=19.8[\mathrm{kN} / \mathrm{m}]$. A bed of lead shot of $2[\mathrm{~mm}]$ diameter is put in the cavity as damper mass. The sinusoidal force is put to $m_{1}$ by electrodynamic sweep shaker through a coil spring $\left(k_{0}=6.6[\mathrm{kN} / \mathrm{m}]\right)$. Four piezo electronic accelerometers with amplifier incorporating integrating circuits are used to measure displacements of main bodies and the shaker table head. Transfer functions in terms of compliance are calculated between response and excitation points by a personal computer.

The effect of the number of damper on the damping performance up to the third resonance is shown in Fig. 8. The dotted curve A represents the measured response without damper, and solid curves B, C and D are the ones for a single, two, and three-unit dampers, respectively, with $d=\infty$, and identical mass ratio $\mu_{d j}\left(=m_{d j} / m_{j}\right)=10 \%$. It can be observed from the figure that a single damper $m_{d 1}$ exerts damping action to the second and third resonant vibrations by virtue of amplification effects associated with corresponding modal amplitudes, while two and threeunit dampers can exert cumulative damping effects to each of the vibration modes. The effect of increasing the number of dampers is prominent for higher resonances except for fundamental one. This is because the damping performance of the particle damper deteriorates with lower acceleration level of main vibrating body.

The approximate SDOF procedure for the damped periodic motion was applied to the 3DOF system with the particle damper for each of its vibration modes. The results of these analyses are compared with those obtained by experiments and simulations. Figure 9 shows the frequency response of the system with a single damper located at $j=2$ for the third mode, when the clearance is optimized such that only Type II motion appears within resonance. The left-hand side ordinate in Fig. 9 is the compliance of the primary mass with and without damper, while the top ordinate represents the impact phase $\varphi$, time of separation from floor $\tau_{0}$, and time of impact at ceiling $\tau_{1}$. In the graph, the approximate SDOF solution is shown as solid curves. 


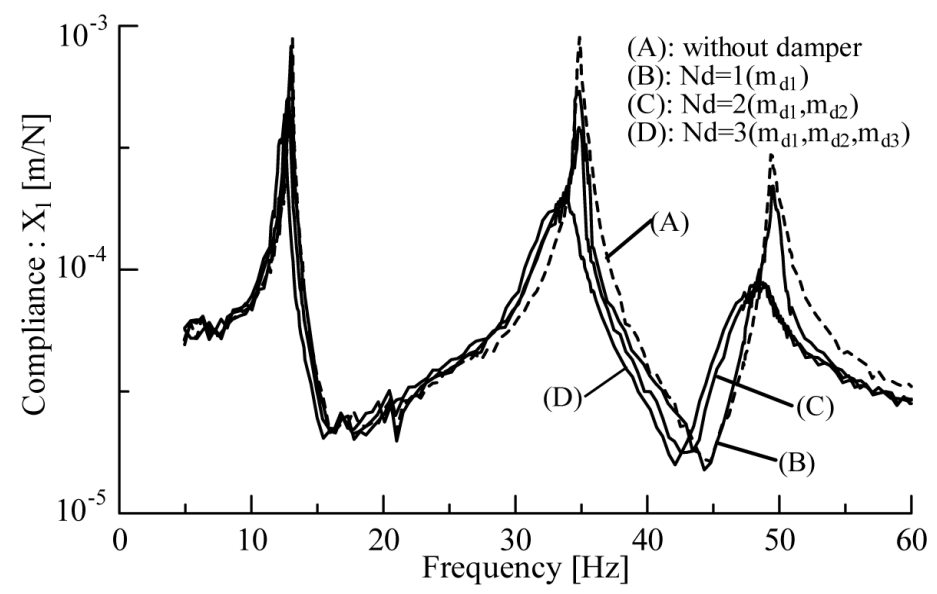

B:

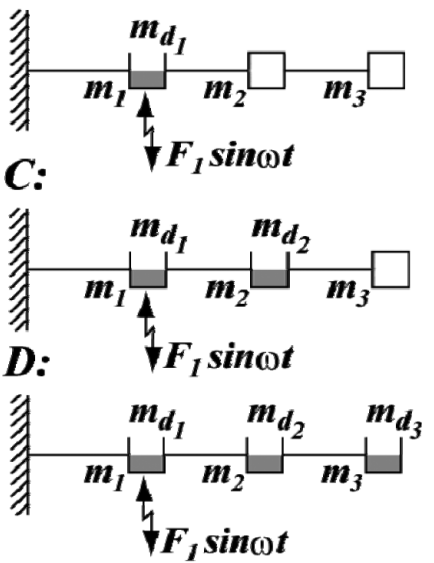

Fig. 8. Measured vibration of frequency response with number of damper unit $N_{d} .\left(\mu_{d j}=10 \%, d_{j}=\infty ; j=1,2,3: F_{1}=2.6[N]\right)$.
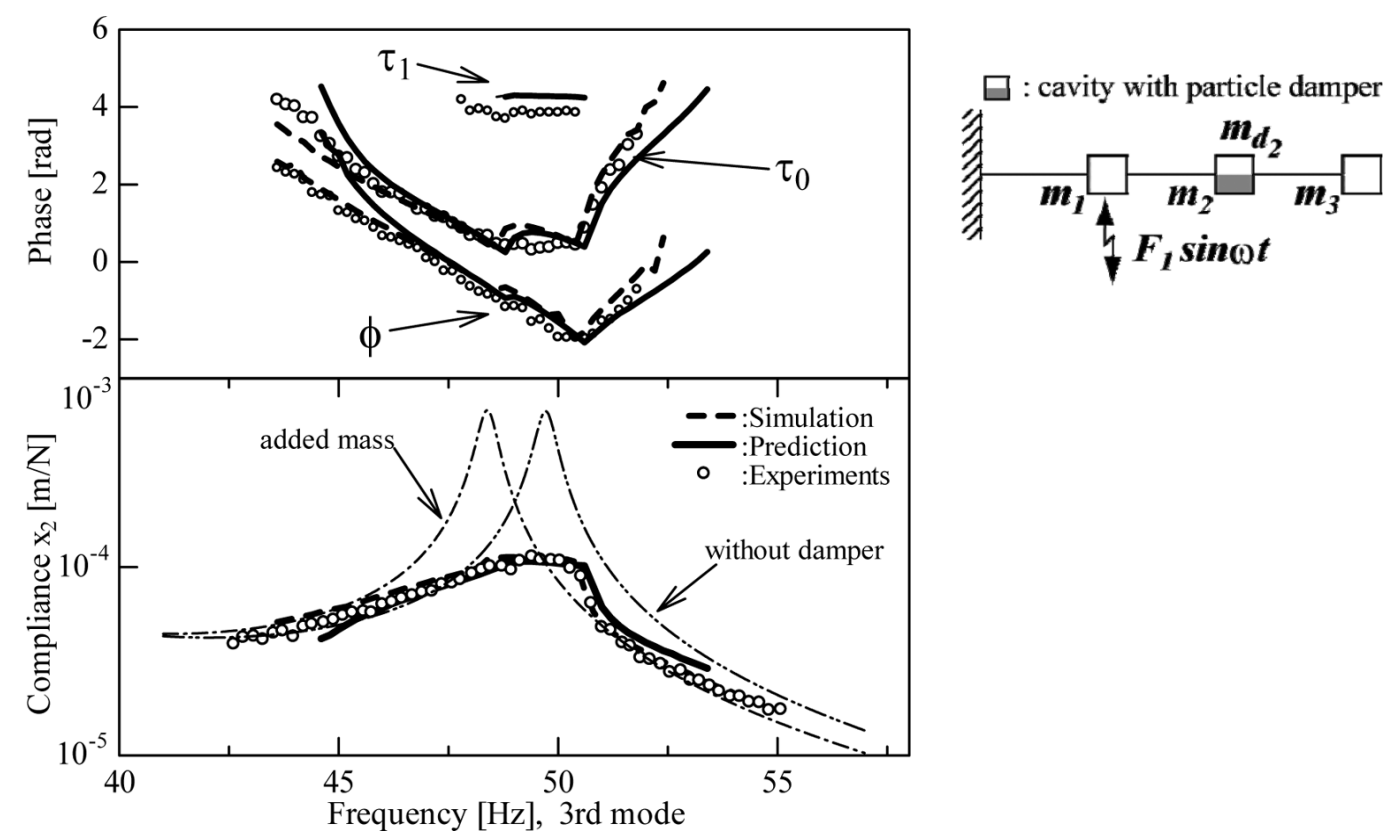

Fig. 9. Comparison between theory, experiment and simulation of 3rd mode response. $\left(\mu_{d 2}=6 \%, d_{2}=0.98[m m], j=1,2,3, F_{1}=\right.$ $3.3[N])$

For the sake of comparison, the graph also contains the two kinds of modal curves of the 3DOF system, one for the absence of the particle damper, the other for the main mass equal to $m_{j}+m_{d j}$ (i.e., with damper added on the primary mass). It is seen that there are generally close agreements between experimental results and those of predictions by the approximate SDOF procedure as well as simulations. Figure 10(a) demonstrates the comparison between simulation and experimental results of the response of the system with mass ratios $\mu_{d j}=m_{d j} / m_{j}=10 \%$, and $d_{j}=\infty(j=1,2,3)$, up to the third resonance, and Fig. 10(b) shows its impulse response of acceleration by experiment. There is generally close agreement between them in the region of resonance except for anti-resonant regions. The differences in these regions are attributed to the deteriorated noise level of the accelerometer used in experiments. In Fig. 10(b), the three units particle damper attenuates the free oscillation faster and more effectively. 


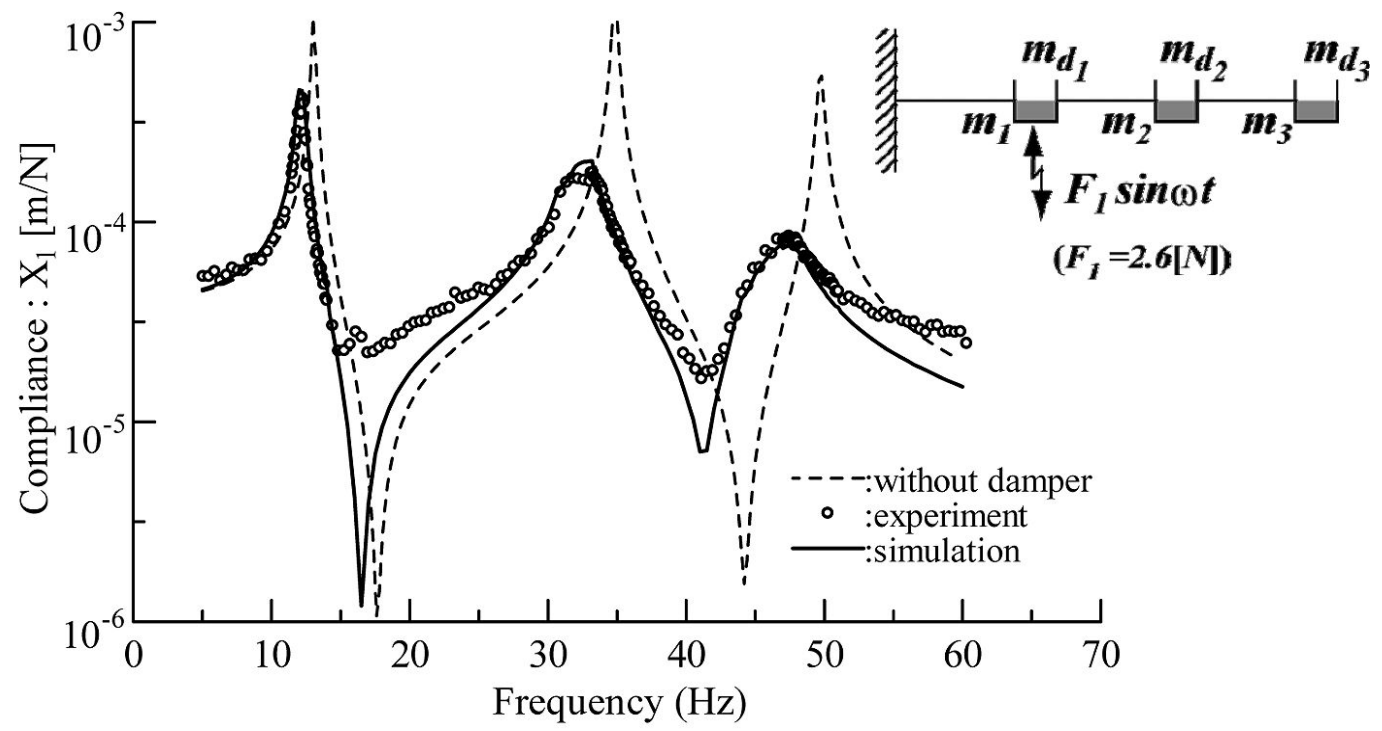

(a) Comparison between experiment and simulation. $(d=\infty)$

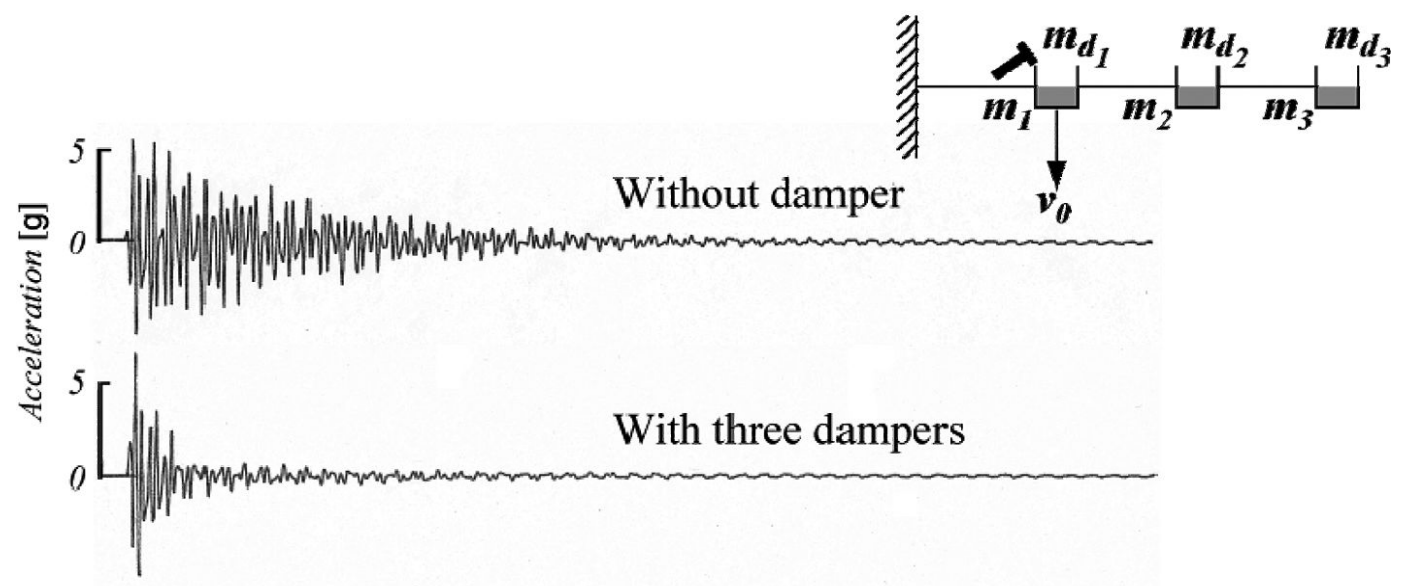

(b) Measured impulse response of acceleration.

Fig. 10. Effectiveness of plural dampers $\left(N_{d}=3\right)$. $\left(\mu_{d j}=10 \%, d_{j}=\infty, j=1,2,3, F_{1}=2.6[N]\right)$.

\section{Summary and conclusions}

An approximate analysis has been presented for determining the damping characteristics of a MDOF system that is provided with the granular particle damper attached to some arbitrary point of the system. The equations of motion are developed for the approximate SDOF system and attached damper masses by means of the normal mode approach, and steady-state solutions to the motion of the MDOF system subjected to the particle damper was derived using SDOF procedure for the periodic motion due to particle damping. Results of the analysis were applied to a 3DOF resonant structure, and the effects of the number of dampers on the damping performance were investigated. It is shown that multi-unit dampers with properly selected mass ratios and clearances effectively suppress the resonant peaks over a wide frequency range. Experimental studies with a 3DOF resonant structure and digital simulations were performed to verify the validity of the analysis. Good correlation was obtained between the theoretical predictions, experiments and simulations. 


\section{References}

[1] H.G. Kaper, The behavior of a mass-spring system provided with a discontinuous dynamic vibration absorber, Applied Science Research Section A 10 (1961), 369-383.

[2] S.F. Masri and T.K. Caughey, On the Stability of the Impact Damper, Transactions of the ASME Journal of Applied Mechanics E 33 (1966), 586-592.

[3] M.I. Feigin, Dynamic Theory of a Controlled Vibration Damping Model, PMM 31(1) (1967), 154-158.

[4] E. Skipor, Application of Impact Damper to Rotary Printing Equipment, ASME Journal of Mechanical Design 102 (1980), $338-343$.

[5] S. Chatterjee, A.K. Mallik and A. Ghosh, On impact dampers for non-linear vibrating systems, Journal of Sound and Vibration 187(3) (1995), 403-420.

[6] M.R. Duncan, C.R. Wassgren and C. M. Krousgrill, The Damping Performance of a Single Particle Impact Damper, Journal of Sound and Vibration 286 (2005), 123-144.

[7] V.B. Stepanov, B.D. Tartakovskii and N. Yu. Fedina, Effectiveness of a filled vibration absorbing coating, Sov Phys Acoust 33(2) (1987), 199-202.

[8] V.B. Stepanov and B.D. Tartakovskii, Statistical method for optimizing the distribution of a vibration absorbing coating on a complex structure, Sov Phys Acoust 35(1) (1989), 69-72.

[9] H.V. Panossian, Structural Damping Enhancement via Non-Obstructive Particle Damping, ASME Journal of Vibration and Acoustics 114 (1992), 101-105

[10] T. Sato, K. Tanaka, S. Aida and Y.Mouri, Vibration Isolation in a System Using granular Medium, JSME International Journal 38 (1995), 434-440.

[11] E.M. Flint, Experimental Measurements of the Particle Damping Effectiveness Under Centrifugal Loads, Proc of the 4th National Turbine Engine High Cycle Fatigue Conference'99 (1999), CD-ROM.

[12] R.D. Friend and V.K. Kinra, Particle Impact Damping, Journal of Sound and Vibration 233(1) (2000), 93-118.

[13] G.R. Tomlinson, Particle Vibration Damper, U.S. Patent, US6547049 B1, 2003.

[14] Y. Wakasawa, M. Hashimoto and E.Marui, The Damping Capacity Improvement of Machine Tool Structures by Balls Packing, International Journal of Machine Tools \& Manufacture 44 (2004), 1527-1536.

[15] Z. Xu, M.Y. Wang and T. Chen, A particle damper for Vibration and noise reduction, Journal of Sound and Vibration 270 (2004), $1033-1040$.

[16] Y. Araki, I. Yokomichi and J. Inoue, Impact Damper with Granular Materials-2nd Report, Both Sides Impact in a Vertical Oscillating System, Bulletin of the JSME 28 (1985), 1466-1472.

[17] X. Fang and J. Tang, Granular Damping in Forced Vibration: Qualitative and Quantitative Analyses, Journal of Vibration and Acoustics 128 (2006), 489-500.

[18] C.J.Wu, W.H.Liao and M.Y.Wang, Modeling of Granular particle Damping using Multiphase Flow Theory of Gas-Particle, ASME Journal of Vibration and Acoustics 126 (2004), 196-201.

[19] M. Saeki, Impact Damping with Granular Materials in a Horizontally Vibrating system, Journal of Sound and Vibration 251(1) (2002), $153-161$.

[20] M. Saeki, Analytical Study of Multi-particle Damping, Journal of Sound and Vibration 281 (2005), $133-1144$.

[21] Z. Xu, M.Y. Wang and T. Chen, An Experimental study of Particle Damping for Beams and Plates, Journal of Vibration and Acoustics 126 (2004), 141-148

[22] Z. Xu, M.Y. Wang and T. Chen, Particle Damping for Passive Vibration Suppression: numerical Modeling and Experimental Investigation, Journal of Sound and Vibration 279 (2005), 1097-1120. 

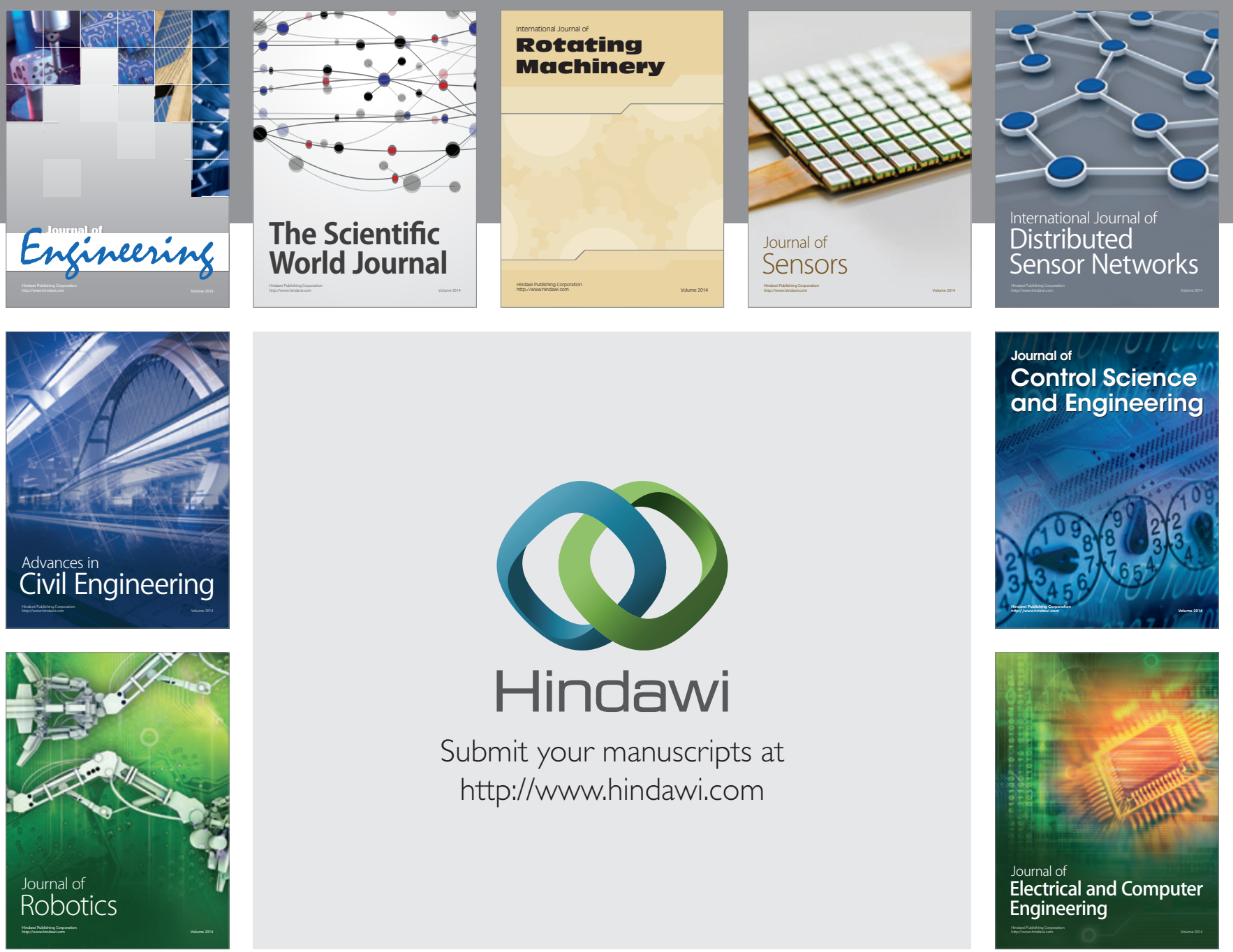

Submit your manuscripts at

http://www.hindawi.com
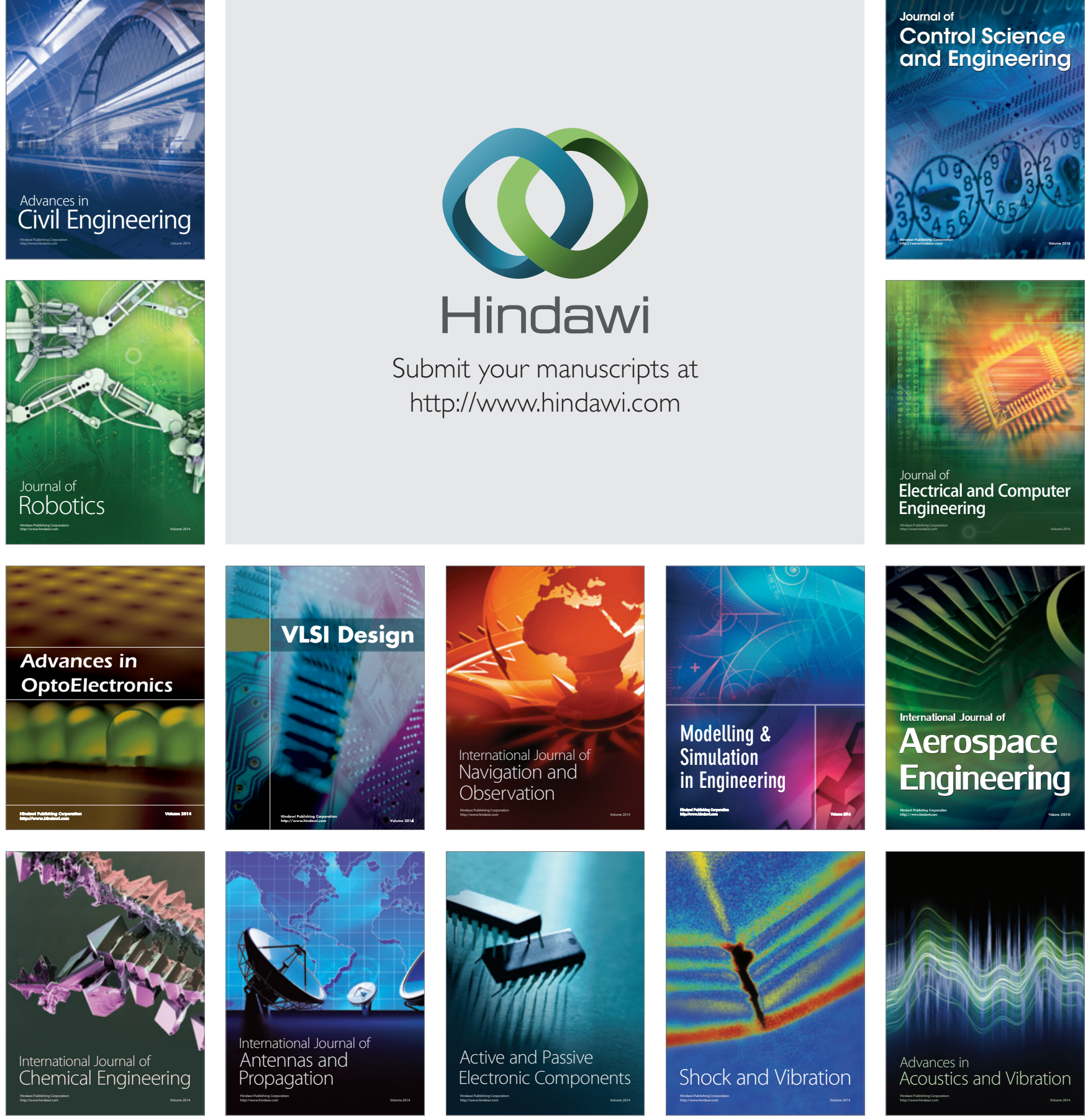